\title{
Overexpression of BNIP3 in Rat Intervertebral Disc Cells Can Trigger Autophagy-Related Apoptosis
}

\section{Ting-Sheng Wu}

Nanchang University

\section{Shao-Yong Fan}

Nanchang Hongdu hospital of Traditional Chinese Medicine

\section{Bin Zhang}

Nanchang Hongdu Hospital of Traditional Chinese Medicine

Zhi-Qiang Tao

Nanchang Hongdu Hospital of Traditional Chinese Medicine

Hong Hua

Nanchang Hongdu Hospital of Traditional Chinese Medicine

Min Dai ( $\square$ daiminqqq89@163.com )

First Affiliated Hospital of Nanchang University https://orcid.org/0000-0002-9719-669X

\section{Research}

Keywords: Bcl-2 and adenovirus E1B 19-kDa interacting protein 3 (BNIP3), intervertebral disc cells, autophagy, apoptosis, intervertebral disc degeneration

Posted Date: July 15th, 2020

DOI: https://doi.org/10.21203/rs.3.rs-42040/v1

License: (1) (1) This work is licensed under a Creative Commons Attribution 4.0 International License.

Read Full License 


\section{Abstract}

Background: Intervertebral disc degeneration (IDD) is the important cause of low back pain. Excessive apoptosis of intervertebral disc cells is the primary cause of IDD. BNIP3 is a member of Bcl-2 family and is widely involved in cell autophagy and apoptosis. However, the roles and mechanisms of BNIP3 in intervertebral disc cell autophagy and apoptosis are unclear.

Results: In this study, the primary rat intervertebral disc cells were prepared and applied to study the effect of BNIP3 overexpression on their autophagy and apoptosis. RT-PCR and western blotting assay showed that BNIP3 overexpression significantly up-regulated the expression of autophagy and pro-apoptotic related proteins, including HIF-1a, Apaf-1, cleaved caspase 3, LC-3 and Beclin-1, while down-regulated the expression of anti-apoptotic protein Bcl-2. Subsequent cell staining detection of autophagy and apoptosis showed that BNIP3 overexpression significantly increased the autophagy and apoptosis of rat intervertebral disc cells. Furthermore, BNIP3 interference assay revealed that the effects of BNIP3 overexpression can be reversed by BNIP3 interference.

Conclusions: The above findings suggested that BNIP3 enhanced autophagy of intervertebral disc cells and further triggered the apoptosis of intervertebral disc cells, eventually led to the development of intervertebral disc degeneration.

\section{Background}

As a common spinal disease, low back pain has become a very serious public health problem in the world, which seriously affecting the physical and mental health of patients and bringing a heavy economic burden to society (1). Although the detailed pathological mechanism of low back pain is elusive up to now, the currently accepted fact is that intervertebral disc degeneration (IDD) is an important cause of low back pain $(2,3)$. The occurrence and development of IDD are related to many factors, such as genetic factors, lifestyle habits (such as smoking or alcohol consumption) and aging. It is worth mentioning that apoptosis of intervertebral disc cells, degradation of extracellular matrix and cell phenotype alterations are still the direct causes of IDD (4). Autophagy is a lysosome-dependent cell degradation pathway that exists exclusively in eukaryotic cells (5). As a type II programmed cell death pathway, autophagy promotes or inhibits apoptosis in different ways. It has reported that autophagy play a crucial role in the development of $\operatorname{IDD}(6,7)$.

$\mathrm{Bcl}-2$ and adenovirus E1B 19-kDa interacting protein 3 (BNIP3) is a member of the BH3-only subfamily of the $\mathrm{Bcl}-2$ family, which is closely related to apoptosis triggered by ischemia and hypoxia (8-10). Present studies suggest that hypoxia-inducible factor-1a (HIF-1a) is the primary regulatory protein of BNIP3 (11), so the expression of BNIP3 is largely upregulated under hypoxic conditions. BNIP3 not only regulate cell apoptosis directly, but also affect the cell survival and apoptosis indirectly by regulating the level of autophagy or mitophagy (12), therefore BNIP3 play vital roles in ischemia or hypoxia related diseases (13-15). Recent studies also show that the BNIP3 expression is positively correlated with the severity of 
IDD, that is, patients with severe IDD usually have higher expression of BNIP3 $(16,17)$, suggest that BNIP3 play an important role in the development of IDD. However, the detailed molecular mechanism of BNIP3 in the development of IDD is unclear.

In this study, the methods of overexpression and RNA interference were used to study the effect of BNIP3 on autophagy and apoptosis in the isolated rat intervertebral disc cells. Our findings showed that overexpression of BNIP3 significantly enhanced autophagy of rat intervertebral disc cells and further led to increased apoptosis, resulted in a decrease in the number of intervertebral disc cells and absence in cell function, which ultimately causes low back pain. Similarly, the opposite results were observed in BNIP3 interference assay. These findings suggested that BNIP3 enhanced autophagy-related apoptosis of intervertebral disc cells and promoted the development of intervertebral disc degeneration.

\section{Results}

\section{Identification of cultured rat intervertebral disc cells}

To study the role of BNIP3 in the autophagy and apoptosis of rat intervertebral disc cells, the primary rat intervertebral disc cells was prepared. The cultured rat intervertebral disc cells were stained with toluidine blue, we found the cytoplasm of the cell was stained to blue and the nucleus was stained to bluish violet (Fig. 1). Furthermore, the cells under the light microscope have the morphological characteristics of intervertebral disc cells, suggested that the isolated and cultured cells were rat intervertebral disc cells.

\section{BNIP3 overexpression decreased the viability of rat intervertebral disc cells}

The transfection method was used to study the effect of BNIP3 on the viability of rat intervertebral disc cells. First, the transfection efficiency in the cells was detected. The expression level of BNIP3 mRNA in BNIP3 overexpression group (oeBNIP3) was increased significantly compared to the overexpression control group (oeControl) or blank group. Similarly, the expression level of BNIP3 mRNA in BNIP3 interference group (siBNIP3) was decreased significantly compared to the interference control group (siControl) or blank group (Fig. 2A). Subsequently, the MTT assay was employed to detect the viability of rat intervertebral disc cells. Our findings showed that although the cell viability in all groups was increased with time, the cell viability of BNIP3 overexpression group was lower than overexpression control or blank group, while BNIP3 interference group had higher cell viability than the interference control or blank group (Fig. 2B). These findings suggested that BNIP3 negatively regulated the viability of rat intervertebral disc cells.

\section{BNIP3 overexpression enhanced the autophagy of rat intervertebral disc cells}


Previously study has reported that BNIP3 could induce autphagy in various types of cells, however, it was unknown that whether BNIP3 could trigger the autophagy of rat intervertebral disc cells. Here, the autophagy of rat intervertebral disc cells was detected by acridine orange staining. We found the rat intervertebral disc cells with BNIP3 overexpression were concentrated and the nuclei were fragmented into dots, and they were stained into uneven, dense and deep stained green particles under light microscope compared to control and interference group (Fig. 3), which was known as the occurrence of autophagy. Similarly, the results of autophagy detected by MDC method also showed that significant autophagy occurred in BNIP3 overexpressed rat intervertebral disc cells, while no obvious autophagy was observed in BNIP3 interference group or control (Fig. 4). The above findings suggested that BNIP3 overexpression triggerred the autophagy of rat intervertebral disc cells.

\section{BNIP3 overexpression or interference disturbed the cell membrane potential}

The cell membrane potential was closely related to cell states such as autophagy and apoptosis. Here, the cell membrane potential of BNIP3 transfected cells was detected by flow cytometry. Our findings showed that the membrane potential of BNIP3 overexpression group was decreased significantly compared to the overexpression control or blank group, while the membrane potential of BNIP3 interference group was increased significantly compared to the interference control or blank group (Fig. 5), suggested that BNIP3 affected the cell membrane potential by triggering autophagy of rat intervertebral disc cells.

\section{BNIP3 induced increased expression of autophagy and apoptosis-related molecules}

In above study, we have observed BNIP3 overexpression could activate the autophagy and apoptosis of rat intervertebral disc cells, and vice versa. Therefore, can BNIP3 affect the expression of autophagy and apoptosis-related proteins? First, the RT-PCR assay was employed to detect the mRNA expression differences of autophagy and apoptosis-related proteins including HIF-1a, Apaf-1, cleaved caspase 3, Bcl2, LC-3 and Beclin-1 in rat intervertebral disc cells after transfected with BNIP3 overexpression or interference vectors. The RT-PCR results showed that the expression of HIF-1a, Apaf-1, cleaved caspase 3, LC-3 and Beclin-1 mRNA was upregulated significantly when the rat intervertebral disc cells transfected with BNIP3 overexpression vector compared to control or blank group. However, the expression of antiapoptosis protein $\mathrm{Bcl}-2$ was decreased in BNIP3 overexpression group compared to the control or blank group. Moreover, the expression of autophagy and apoptosis-related proteins in rat intervertebral disc cells after BNIP3 RNAi was also detected by RT-PCR, the results showed that the expression of HIF-1a, Apaf-1, cleaved caspase 3, LC-3 and Beclin-1 was decreased significantly when the rat intervertebral disc cells transfected with BNIP3 interference vector compared to control or blank group. Similarly, the 
expression of anti-apoptosis protein Bcl-2 was increased in BNIP3 interference group compared to the control or blank group (Fig. 6).

In addition, western blotting analysis of autophagy and apoptosis-related proteins revealed that the expression of HIF-1a, Apaf-1, cleaved caspase 3, LC-3 and Beclin-1 was increased when the rat intervertebral disc cells transfected with BNIP3 overexpression vector compared to the overexpression control and blank group. Interestingly, the expression of anti-apoptosis protein Bcl-2 was decreased in BNIP3 overexpression group compared to the control or blank group. In addition, the BNIP3 RNAi assay revealed that the expression of HIF-1a, Apaf-1, cleaved caspase 3, LC-3 and Beclin-1 was decreased significantly when the rat intervertebral disc cells transfected with BNIP3 interference vector compared to control or blank group. Similarly, the expression of anti-apoptosis protein Bcl-2 was increased in BNIP3 interference group compared to the control or blank group (Fig. 7A). To better display the expression differences of HIF-1a, Apaf-1, cleaved caspase 3, Bcl-2, LC-3 and Beclin-1among different groups, the gray scale analysis of western blotting bands was performed and the similar results were obtained (Fig. 7B). These findings indicated that BNIP3 activate the expression of autophagy and apoptosis-related proteins and finally lead to the occurrence of autophagy and apoptosis of rat intervertebral disc cells.

Taken together, our findings showed that BNIP3 triggered autophagy and apoptosis of rat intervertebral disc cells via stimulating the expression of autophagy and apoptosis-related proteins. The augment of autophagy and apoptosis of rat intervertebral disc cells led to development of intervertebral disc degeneration and finally exacerbated the process of low back pain.

\section{Discussion}

Apoptosis of lumbar intervertebral disc cells is one of the important causes of $\operatorname{IDD}(4,20)$. Numerous studies have shown that the apoptosis rate of nucleus pulposus cells in IDD patients or IDD model animals is significantly higher than that in nucleus pulposus cells of normal humans or animals (4). In addition, many factors that can induce IDD such as ischemia and hypoxia, nutritional deficiencies, stress load, high glucose and hypertonicity, not only activated autophagy but also induced apoptosis (21-23). The present perspective believed that autophagy can inhibit apoptosis by clearing damaged cells, but excessive autophagy can also induce autophagic apoptosis $(24,25)$. Autophagy is a lysosomedependent catabolism pathway that exclusively exists in eukaryotic cells and composed of a series of autophagy-related genes, which can degrade and recycle damaged, senescent and denatured organelles or macromolecular substances in cells (26). Finally, autophagy provides sufficient energy for cell survival. Autophagy mainly includes macrophage, microautophagy and molecular chaperone-mediated autophagy. The process of autophagy mainly divides into five stages: autophagy induction, autophagy vesicle nucleation, extension, autophagosome formation and autophagy lysosome formation (27). It has reported that autophagy played many different important roles in the development of IDD. Ye et al. (28) found that the number of autophagosomes and the ratio of autophagy-related proteins LC-3II/LC-3I in rat intervertebral disc nucleus pulposus cells increased with the age of rats. Xu et al. (29) found that the autophagy level of endplate cells in IDD patients was lower than that in normal humans, while Gruber et 
al. (30) showed that the autophagy level of the annulus fibrosus in patients with IDD was higher than that in normal humans. The above studies indicated it's discriminative on the exact roles of autophagy in IDD. One of the possible reasons was that the patients adopted in these studies have different severity of intervertebral disc degeneration. The other possible reason was the tissues used were from different sites. However, the detailed role and molecular mechanisms of autophagy in the development of IDD were unclear. Therefore, present study aimed to explore the role and mechanisms of autophagy in the apoptosis of rat intervertebral disc cells.

The BNIP3 gene is a protein discovered by Boyd and other scholars in 1994 through yeast hybridization technology that can interact with the adenovirus E1B19kD protein and can encode 194 amino acids. The encoded protein has two dimers. Subsequent studies confirmed that BNIP3 is a pro-apoptotic protein of the Bcl-2 family (31). Its C-terminus can bind to the mitochondrial membrane, which increases the permeability of the mitochondrial membrane and reduces the potential of the mitochondrial membrane, which in turn promotes apoptosis (32). The biological role of BNIP3 under hypoxic conditions mainly depends on the regulation of HIF-1a, which can bind to the HRE response element of the BNIP3 promoter, thereby regulating the transcription of BNIP3. Therefore, scholars believe that the hypoxic environment can significantly induce the expression of BNIP3, and the moderate expression of BNIP3 can activate autophagy and maintain the survival of cells, while excessive autophagy causes apoptosis (13-15). Current research showed that the positive expression rate of BNIP3 gradually increases with the severity of IDD, and the expression of BNIP3 was closely related to the apoptosis of intervertebral disc cells during the development of IDD (16). Previous studies showed that BNIP3 play important roles in the development of IDD, but the detailed mechanism was elusive.

In this study, the BNIP3 overexpression and RNA interference methods were employed to explore the role and mechanisms of BNIP3 in IDD process. First, the success of transfection was verified by RT-PCR. Second, the CCK-8 method was used to detect the cell viability of rat intervertebral disc cells after transfected with different vectors and revealed that BNIP3 overexpression remarkable decreased the cell viability and BNIP3 interference remarkable increased the cell viability (Fig. 2B). These findings were consistent with the evidence that BNIP3 closely related with the apoptosis of intervertebral disc cells in IDD development reported previously (33), suggested that BNIP3 expression significant affected the viability of rat intervertebral disc cells. Subsequently, the autophagy of rat intervertebral disc cells detected by acridine orange staining and MDC staining methods, the findings showed that BNIP3 overexpression increased the autophagy and BNIP3 interference decreased the autophagy of rat intervertebral disc cells (Figs. 3 and 4). Flow cytometry detection of cell membrane potential revealed that BNIP3 overexpression significant decreased the membrane potential while BNIP3 interference increased the membrane potential of rat intervertebral disc cells (Fig. 5), suggested that BNIP3 activated autophagy related apoptosis and further induced a decreased number of intervertebral disc cells, eventually led to the occurrence of IDD.

Cell apoptosis and autophagy are closely associated with apoptosis- or autophagy-related proteins and pathways. Bcl-2 is one of the most important anti-apoptosis proteins that can form a dimer with the pro- 
apoptotic protein Bax, then inhibit apoptosis signaling and promote cell survival (34). Beclin-1 is an autophagy-related gene that is homologous with yeast autophagy gene 6 . Beclin- 1 can recruit other autophagy-related proteins to localize on the membrane of autophagosome, which plays a positive regulatory role on the formation of autophagosomes (35). Furthermore, Beclin-1 also interacts with Bcl-2 through its $\mathrm{BH} 3$ domain to maintain the dynamic equilibrium of cell autophagy and apoptosis (36). LC3 is the homolog of mammalian autophagy gene 8 that contains two forms (LC-3I and LC-3II), especially the content of LC-3II to a certain extent reflects the cell's autophagy activity. BNIP3 can competitively bind to $\mathrm{Bcl}-2$ with Beclin-1 because both of them contains $\mathrm{BH} 3$ domain. The overexpression of BNIP3 causes the release of Beclin-1 from the Beclin-1/Bcl-2 complex. The free Beclin-1 then forms a complex with PI3K and further regulates the expression of various autophagy-related genes downstream of PI3K/AKT, eventually promote the occurrence of mitochondrial autophagy $(37,38)$. In addition, the BNIP3 overexpression increases the mitochondrial membrane permeability and membrane potential, then leads to the release of cytochrome $C$. The binding of cytochrome $C$ with Apaf- 1 can activate caspase pathway, then induces a series of cascades and triggers apoptosis. In this study, RT-PCR and western blotting methods were employed to study the expression differences of autophagy and apoptosis-related proteins of rat intervertebral disc cells after transfected with BNIP3 overexpression or interference vectors. Our findings showed that the expression of pro-apoptotic or autophagic proteins such as HIF-1a, Apaf-1, cleaved caspase 3, LC-3 and Beclin-1 were increased significantly after the cells overexpressed with BNIP3, while the expression of anti-apoptotic protein such as Bcl-2 was decreased significantly (Figs. 6 and 7). Similarly, the expression of pro-apoptotic or autophagic proteins such as HIF-1a, Apaf-1, cleaved caspase 3, LC-3 and Beclin-1 were decreased significantly after the cells transfected with BNIP3 terference vector, while the expression of anti-apoptotic protein such as Bcl-2 was increased significantly (Figs. 6 and 7). These findings suggested that BNIP3 overexpression increased the autophagy-related apoptosis of intervertebral disc cells by regulating the expression of autophagy- and apoptosis-related proteins, thereby triggered autophagic apoptosis.

In conclusion, BNIP3 overexpression triggered autophagic apoptosis by activating the expression of autophagy- and apoptosis-related proteins in intervertebral disc cells, then decreased the viability of intervertebral disc cells and led to the occurrence of intervertebral disc degeneration, caused low back pain.

\section{Conclusions}

In this study, the primary rat intervertebral disc cells were prepared and applied to study the effect of BNIP3 overexpression on their autophagy and apoptosis. Our findings showed that BNIP3 overexpression in rat intervertebral disc cells significantly up-regulated the expression of autophagy and pro-apoptotic related proteins, and then enhanced the autophagy and apoptosis of rat intervertebral disc cells. Moreover, the effects of BNIP3 overexpression could be reversed significantly by BNIP3 interference. These findings indicated that BNIP3 enhanced autophagy of intervertebral disc cells and further triggered the apoptosis of intervertebral disc cells, eventually led to the development of intervertebral disc degeneration. 


\section{Materials And Methods}

\section{Animals}

20 healthy adult Sprague-Dawley (SD) rats weighting 200 to $250 \mathrm{~g}$ were used in this study. The animals were purchased from Beijing Charles River Laboratory Animal Co., Ltd. The rats were housed in individual cages under a constant temperature $\left(23 \pm 2{ }^{\circ} \mathrm{C}\right)$ and had access to food and water ad libitum throughout the study. All procedures, care, and handling of the rats were approved by the Ethics Committee of Nanchang Hongdu hospital of traditional Chinese medicine.

\section{Antibodies and Reagents}

The SuperSignal ${ }^{\text {TM }}$ West Pico PLUS chemiluminescent substrate (34580) was purchased from Thermo Fisher Scientific Inc. (Waltham, MA, USA). PVDF membrane with $0.45 \mu \mathrm{m}$ pore size (IPVH00010) was purchased from Merck Millipore (Darmstadt, Germany). BCA protein assay kit (CW0014S), HiFiScript first strand cDNA synthesis kit (CW2569M), UltraSYBR mixture (CW0957M), Ultrapure RNA extraction kit (CW0581M) and TRIzon reagent (CW0580S) were purchased from Cwbio Inc (Beijing, China). Mouse antiGAPDH monoclonal antibody (TA-08), HRP-conjugated goat anti-mouse IgG $(\mathrm{H}+\mathrm{L})$ secondary antibody (ZB-2305) and HRP-conjugated goat anti-rabbit IgG $(\mathrm{H}+\mathrm{L})$ secondary antibody (ZB-2301) were purchased from ZSGB-Bio Technologies Inc (Beijing, China). Rabbit anti-HIF-1 a polyclonal antibody (ab2185), rabbit anti-Bcl-2 polyclonal antibody (ab59348), rabbit anti- Apaf-1 monoclonal antibody (ab32372), rabbit anti-cleaved caspase 3 polyclonal antibody (ab2302) and rabbit anti- Beclin-1 polyclonal antibody (ab62557) were purchased from Abcam (Cambridge, UK). The rabbit anti-LC3 polyclonal antibody (bs-8878R) was purchased from Biosynthesis Inc (Beijing, China). Cell autophagy staining test kit (G0170) and Acridine orange staining solution (CA1143) were purchased from Solarbio Life Sciences (Beijing, China). All other reagents were purchased from Sigma-Aldrich (St. Louis, MO, USA).

\section{Isolation and culture of rat intervertebral disc cells}

Twenty SD rats were used to isolate the intervertebral disc cells. In brief, the rats were anesthetized and killed by intraperitoneal injection of $10 \%$ chloral hydrate, then isolate the spine under aseptic conditions, following removal of ligaments and nucleus pulposus around the spine. The isolated spine was cut into small block with the size of $1 \mathrm{~mm} 3$, after digestion with $0.25 \%$ trypsin for $20 \mathrm{~min}$ and $0.2 \%$ type II collagenase digestion for $2 \mathrm{~h}$, the digested cells were collected by cell sieve and cultured in the DMEM/F12 medium containing $10 \%$ fetal bovine serum, $100 \mathrm{U} / \mathrm{ml}$ penicillin and $100 \mu \mathrm{g} / \mathrm{ml}$ streptomycin under an atmosphere of $5 \% \mathrm{CO} 2$ at $37{ }^{\circ} \mathrm{C}$, the cultured medium was changed every $2-3$ days. When the degree of cell fusion reached more than $80 \%$, digested the cells at a ratio of $1: 2$ for passage. At the same time, the second generation cells were taken for the detection of toluidine blue staining. 


\section{Toluidine blue staining}

The above cultured rat intervertebral disc cells grown to the second generation, then inoculated them on 6-well plate, removed the medium and washed with phosphate buffer solution (PBS) for 5 min, then the cells fixed with $4 \%$ paraformaldehyde at room temperature for $10 \mathrm{~min}$. Subsequently, the fixation solution was removed and the cells were stained with toluidine blue dye for $2 \mathrm{~min}$, the staining solution was removed and the cells were washed with ultrapure water for $3 \mathrm{~min}$. The stained and washed cells then air dried and imaged under optical microscope.

\section{Overexpression and RNA interference assay}

To study the role of BNIP3 in the autophagy and apoptosis of rat intervertebral disc cells, the overexpression and RNA interference assay were performed as described previously (18). Briefly, the rat intervertebral disc cells were placed in 6-well tissue culture plates and growth to $60 \%$ confluence prior to transfection. Then the cells were transfected with $4 \mu \mathrm{g}$ of plasmids (built recombinant BNIP3-pcDNA 3.1 vector and pcDNA 3.1 empty vector) for $48 \mathrm{~h}$. The Lipofectamine 3000 transfection reagent was used. For RNA interference (RNAi) experiments, the cells were growth to $40-50 \%$ confluence prior to transfection. The recombinant BNIP3 silenced plasmid sh-BNIP3-pLVshRNA (siBNIP3) and control vector (siCtrl) were transfected into cells by using the Lipofectamine 3000 transfection reagent for $48 \mathrm{~h}$. After BNIP3 overexpression and RNAi, the expressions of BNIP3 mRNA in rat intervertebral disc cells were detected by real-time quantitative PCR.

\section{Cell viability determination by CCK8 method}

To determine the viability of rat intervertebral disc cells after tranfected with BNIP3 overexpression vector or interference vector, the CCK8 method was employed. Briefly, the transfected cells were placed in 96-well plates and added $150 \mu \mathrm{L}$ freshly prepared toxicity test solution containing $10 \mu \mathrm{L}$ CCK8 to each well and cultured for $4 \mathrm{~h}$, the absorbance at $450 \mathrm{~nm}$ wavelength in $0 \otimes 12 \varangle 24$ and $72 \mathrm{~h}$ were measured by microplate reader. The cell viability assay was repeated in three times and the data was presented as Means \pm SD. The cell growth inhibition rate $(\%)=(O D 450$ Control - OD450Experiment $) /$ OD450Control $\times 100 \%$. The cell growth inhibition rate curve was drawed, the groups as abscissa and cell growth inhibition rate (\%) as ordinate.

\section{Cell autophagy determination by acridine orange staining}

The above transfected cells were collected and prepared to cell suspension at the concentration of $1 \times$ $106 \mathrm{cells} / \mathrm{ml}$. Every $100 \mu \mathrm{L}$ cell suspension mixed with acridine orange staining solution with a final concentration of $15 \mu \mathrm{g} / \mathrm{mL}$, stained at room temperature in the dark for $20 \mathrm{~min}$, then drop it on a glass slide and observed under fluorescent microscope. The normal cells were presented to uniform yellow- 
green fluorescence, while autophagic cells' chromatins were concentrated and the nuclei were fragmented into dots, and they were stained into uneven, dense and deep stained green particles.

\section{Cell autophagy determination by MDC method}

The transfected cells collected by trypsin digestion and washed with PBS, then suspended in $1 \times$ wash buffer with the concentration of $1 \times 106 \mathrm{cells} / \mathrm{ml}$. $90 \mu \mathrm{L}$ cell suspension was taken out and transferred to new eppendorf tube. Whereafter, $10 \mu \mathrm{L}$ MDC dye was added into the cell suspension, mixed and stained at room temperature in the dark for $45 \mathrm{~min}$, the stained cells were collected by centrifuged. The cells were washed two times with $1 \times$ wash buffer and resuspended in $100 \mu \mathrm{L}$ collection buffer. The cell suspension was loaded on the slide and observed under fluorescent microscope. The normal cells were presented to uniform yellow-green fluorescence, while autophagic cells' chromatins were concentrated and the nuclei were fragmented into dots, and they were stained into uneven, dense and deep stained green particles.

\section{Cell membrane potential was detected by flow cytometry}

The membrane potential detection of transfected cells by using flow cytometry method. Briefly, the transfected cells collected by trypsin digestion and washed two times with PBS, then suspended in JC-1 working buffer with the concentration of $1 \times 106 \mathrm{cells} / \mathrm{ml}$. The cell suspensions incubated at $37^{\circ} \mathrm{C}$ for 20 min, centrifuged, collected the cells and resuspended in $1 \times$ Incubation buffer. Finally, the cells were analyzed by using a flow cytometer.

\section{Real-time fluorescent quantitative PCR (RT-PCR)}

Real-time fluorescent quantitative PCR was used to detect the expression of HIF-1a, Bcl-2, Apaf-1, cleaved caspase 3, LC-3 and Beclin-1 mRNA of transfected rat intervertebral disc cells. In brief, the total RNA of transfected cells was extracted by commercial RNA extraction kit and the RNA purity were determined by the ratio of A260/A280 and agarose gel electrophoresis. Total RNA was synthesized the first strand cDNA by first strand cDNA synthesis kit. Using the synthesized first-strand cDNA as template, the specific primers for HIF-1a, Bcl-2, Apaf-1, cleaved caspase 3, LC-3 and Beclin-1 were designed as the previous method (19) and listed on Table 1 and applied to RT-PCR reaction. The RT-PCR reaction condition as follows: $95^{\circ} \mathrm{C}$ for $10 \mathrm{~min}, 95^{\circ} \mathrm{C}$ for 5 and $60^{\circ} \mathrm{C}$ for $1 \mathrm{~min} \otimes 40$ cycles. The RT-PCR reaction system was 20 $\mu \mathrm{L}$, which contained $2 \mu \mathrm{L}$ cDNA template, $0.8 \mu \mathrm{L}$ the primer $\mathrm{F}$ and primer $\mathrm{R}, 10 \mu \mathrm{L}$ SYBR Green solution and $7.2 \mu \mathrm{L} \mathrm{ddH} 2 \mathrm{O}$. The data of RT-PCR was calculated and analyzed by the method of $2-\Delta \Delta \mathrm{CT}$. 
Table 1

Sequences of primers used for detection of BNIP3, Beclin-1, LC-3, Bcl-2, HIF-1 a, caspase 3, Apaf1 and GAPDH in rat.

\begin{tabular}{|c|c|c|c|}
\hline Primer name & $\begin{array}{l}\text { Primer sequence } \\
\text { (F: Forward, R: Reverse) }\end{array}$ & Primer length(bp) & Product length(bp) \\
\hline \multirow[t]{2}{*}{ BNIP3 } & F: TCTCACTGTGACAGCCCACC & 20 & \multirow[t]{2}{*}{183} \\
\hline & R: CCGACTTGACCAATCCCATA & 20 & \\
\hline \multirow[t]{2}{*}{ Beclin-1 } & F: GGAGAAAGGCAAGATTGAAGA & 21 & \multirow[t]{2}{*}{140} \\
\hline & R: AGGACACCCAAGCAAGACC & 19 & \\
\hline \multirow[t]{2}{*}{ LC-3 } & F: ATGGCGGCGTCTTTGTG & 17 & \multirow[t]{2}{*}{310} \\
\hline & R: TGGATTTCTTCAGTTGCTTGG & 21 & \\
\hline \multirow[t]{2}{*}{$\mathrm{Bcl}-2$} & F: CCCTGGCATCTTCTCCTTC & 19 & \multirow[t]{2}{*}{269} \\
\hline & R: AGAGTTCCTCCACCACCGT & 19 & \\
\hline \multirow[t]{2}{*}{ HIF-1a } & F: GCCCTGGATGGCTTTGT & 17 & \multirow[t]{2}{*}{200} \\
\hline & R: TGTTCTTTCCССTTTCTCACT & 21 & \\
\hline \multirow[t]{2}{*}{ Caspase 3} & F: AAAGCCGAAACTCTTCATCA & 20 & \multirow[t]{2}{*}{63} \\
\hline & R: GTCTCAATACCGCAGTCCAG & 20 & \\
\hline \multirow[t]{2}{*}{ Apaf-1 } & F: TCCATCGGAAAACAAGACAA & 20 & \multirow[t]{2}{*}{144} \\
\hline & R: TCGCAGCATCAGAACACG & 18 & \\
\hline \multirow[t]{2}{*}{ GAPDH } & F: GCAAGTTCAACGGCACAG & 18 & \multirow[t]{2}{*}{141} \\
\hline & R: CGCCAGTAGACTCCACGAC & 19 & \\
\hline
\end{tabular}

\section{Western blotting}

Western blotting was used to detect the expression level of HIF-1a, Bcl-2, Apaf-1, cleaved caspase 3, LC-3 and Beclin-1 proteins of transfected rat intervertebral disc cells. Briefly, the cells were collected and washed, then lysed by adding RIPA lysate contained protease inhibitor cocktail in ice-bath for $20 \mathrm{~min}$. The above cell lysate was centrifuged at $10000 \mathrm{rpm}$ for $15 \mathrm{~min}$, the supernatant after centrifugation is the total proteins. The concentrations of proteins were determined by BCA method. The total proteins were loaded and separated by $12 \%$ SDS - PAGE, then electrotransferred onto PVDF membranes. The PVDF membranes were subsequently blocked with $3 \%$ BSA and subsequently incubated with rabbit anti-HIF-1a (1:1000 dilution), anti-Bcl-2 (1:1000 dilution), anti-Apaf-1 (1:1000 dilution), anti-cleaved caspase 3 (1:1000 dilution), anti-LC-3 (1:500 dilution), anti-Beclin-1 (1:1000 dilution) polyclonal antibody and mouse 
anti-GAPDH monoclonal antibody (1:10000 dilution), respectively. The incubation of PVDF and primary antibodies was overnight at $4{ }^{\circ} \mathrm{C}$. After incubated with primary antibodies, the PVDF membrane washed with TBS buffer contained 1\%o Tween-20 three times, then incubated with HRP-conjugated goat antirabbit or goat anti-mouse secondary antibody (1:5000 dilution). Finally, the protein bands were visualized with the SuperSignal ${ }^{\mathrm{TM}}$ West Pico PLUS chemiluminescent substrate.

\section{Statistical analysis}

All experimental values were presented as the Mean \pm SD. Each experiment was repeated at least 3 times independently. Statistical analysis of data was performed using SPSS 17.0 software. One-way ANOVA was used to analyse the differences between more than two groups, and non-paired student's $t$ test was used to analyse the difference between two groups. $\mathrm{P}<0.05$ was considered statistically significant.

\section{Declarations}

\section{Ethical Approval and Consent to participate}

Not applicable

\section{Consent for publication}

Not applicable

\section{Availability of supporting data}

Not applicable

\section{Competing interests}

The authors declare that they have no competing interests.

\section{Funding}

Not applicable

\section{Authors' contributions}

M.D. and T.W. conceived and designed the experiments and analyzed results. S.F., B.Z., Z.T., and H.H. carried out most of the experiments, including western blotting detection, flow cytometry analysis and 
animal experiments. T.W., S.F. and B.Z. participated in data analysis. T.W. and M.D. wrote the paper. Z.T. and $\mathrm{H}$.H. edited the manuscript.

\section{Acknowledgements}

Not applicable

\section{References}

1. Edward J, Carreon LY, Williams MV, Glassman S, \& Li J (2018) The importance and impact of patients' health literacy on low back pain management: a systematic review of literature. Spine $J$ 18(2):370-376.

2. Le Maitre CL BA, Thorpe AA, Hughes SP. (2015) Degeneration of the intervertebral disc with new approaches for treating low back pain. J Neurosurg Sci 59(1):47-61.

3. Roquelaure APY (2015) Low Back Pain, Intervertebral Disc and Occupational Diseases. Int J Occup Saf Ergon 21(1):15-19.

4. Wu T-tXFLH-tJP-jTL-wXaC-I (2015) Research advance on intervertebral disc degeneration and cell death. China J Orthop Trauma 28(7):673-678.

5. Maiuri MC, Zalckvar E, Kimchi A, \& Kroemer G (2007) Self-eating and self-killing: crosstalk between autophagy and apoptosis. Nature Reviews Molecular Cell Biology 8(9):741-752.

6. Yurube T, Ito M, Kakiuchi Y, Kuroda R, \& Kakutani K (2020) Autophagy and mTOR signaling during intervertebral disc aging and degeneration. JOR Spine 3(1):e1082.

7. Zhang S-J, et al. (2016) Autophagy: A double-edged sword in intervertebral disk degeneration. Clinica Chimica Acta 457:27-35.

8. Azad MB, et al. (2008) Hypoxia induces autophagic cell death in apoptosis-competent cells through a mechanism involving BNIP3. Autophagy 4(2):195-204.

9. Lori A. Kubasiak OMH, Nanette H. Bishopric, and Keith A. Webster (2002) Hypoxia and acidosis activate cardiac myocyte death through the Bcl-2 family protein BNIP3. Proc Natl Acad Sci. USA 99(20):12825-12830.

10. Hamacher-Brady A, et al. (2007) Response to myocardial ischemia/reperfusion injury involves Bnip3 and autophagy. Cell Death Differ 14(1):146-157.

11. Heidi M. Sowter PJR, Peter Watson, Arnold H. Greenberg, and Adrian L. Harris (2001) HIF-1-dependent Regulation of Hypoxic Induction of the Cell Death Factors BNIP3 and NIX in Human Tumors. Cancer Research 61(18):6669-6673.

12. Zhang T, et al. (2016) BNIP3 Protein Suppresses PINK1 Kinase Proteolytic Cleavage to Promote Mitophagy. J Biol Chem 291(41):21616-21629.

13. Choi $\mathrm{H}$, et al. (2016) Hypoxia promotes noncanonical autophagy in nucleus pulposus cells independent of MTOR and HIF1A signaling. Autophagy 12(9):1631-1646. 
14. Nagarjuna Vasagiri VKK (2014) Structure, Function, and Epigenetic Regulation of BNIP3: A Pathophysiological Relevance Mol Biol Rep 41(11):7705-7714.

15. Ney PA (2015) Mitochondrial autophagy: Origins, significance, and role of BNIP3 and NIX. Biochim Biophys Acta 1853(10 Pt B):2775-2783.

16. Jie Liu NP, Yan Lu, Jian Wang (2012) Preliminary research for correlation between BNIP3 expression and disc degeneration. Chinese Journal of Spine and Spinal Cord 22(1):65-71.

17. Jie Liu NP, Jian Wang (2012) Immunohistochemistry research on BNIP3 expressions of degenerated intervertebral disc in rabbit. J REG ANAT OPER SURG 21(1):3-5.

18. Guo XL, et al. (2019) Endogenous pore-forming protein complex targets acidic glycosphingolipids in lipid rafts to initiate endolysosome regulation. Commun Biol 2:59.

19. Ye Q, et al. (Giardia's primitive GPL biosynthesis pathways with parasitic adaptation 'patches': implications for Giardia's evolutionary history and for finding targets against Giardiasis. Scientific Reports 7(1):9507.

20. Zhang F, Zhao X, Shen H, \& Zhang C (2016) Molecular mechanisms of cell death in intervertebral disc degeneration (Review). Int J Mol Med 37(6):1439-1448.

21. Xueqiang Shen HJ (2017) Research progress on the relationship between autophagy and intervertebral disc degeneration. Chinese J Trad Med Traum \& Orthop 25(8):77-82.

22. Peng Ao C-cY, Tian-long Wu, Wen-zhou Huang, Xi-gao Cheng (2016) Progress in Association between Autophagy and Intervertebral Disc Degeneration. Chinese Journal of Cell Biology 38(11):1382-1390.

23. De-Heng Chen K-LZ, Hua-Zi Xu (2016) Research progress on the role of autophagy in intervertebral disc degeneration. J Med Res 45(12):9-12.

24. Ding F, Shao ZW, \& Xiong LM (2013) Cell death in intervertebral disc degeneration. Apoptosis 18(7):777-785.

25. Chen JW, et al. (2014) The responses of autophagy and apoptosis to oxidative stress in nucleus pulposus cells: implications for disc degeneration. Cell Physiol Biochem 34(4):1175-1189.

26. Mizushima N (2007) Autophagy: process and function. Genes Dev 21(22):2861-2873.

27. Yorimitsu T \& Klionsky DJ (2005) Autophagy: molecular machinery for self-eating. Cell Death Differ 12 Suppl 2:1542-1552.

28. Ye W, et al. (2011) Age-related increases of macroautophagy and chaperone-mediated autophagy in rat nucleus pulposus. Connect Tissue Res 52(6):472-478.

29. Xu H, Xiong S, Wang H, Zhang M, \& Yu Y (2014) The evidence and the possible significance of autophagy in degeneration model of human cervical end-plate cartilage. Exp Ther Med 7(3):537-542.

30. Gruber HE, Hoelscher GL, Ingram JA, Bethea S, \& Hanley EN, Jr. (2015) Autophagy in the Degenerating Human Intervertebral Disc: In Vivo Molecular and Morphological Evidence, and Induction of Autophagy in Cultured Annulus Cells Exposed to Proinflammatory CytokinesImplications for Disc Degeneration. Spine (Phila Pa 1976) 40(11):773-782. 
31. Burton TR \& Gibson SB (2009) The role of Bcl-2 family member BNIP3 in cell death and disease: NIPping at the heels of cell death. Cell Death Differ 16(4):515-523.

32. Zhang J \& Ney PA (2009) Role of BNIP3 and NIX in cell death, autophagy, and mitophagy. Cell Death Differ 16(7):939-946.

33. Liu J, Wang J, \& Zhou Y (2012) Upregulation of BNIP3 and translocation to mitochondria in nutrition deprivation induced apoptosis in nucleus pulposus cells. Joint Bone Spine 79(2):186-191.

34. Willis S, Day CL, Hinds MG, \& Huang DC (2003) The Bcl-2-regulated apoptotic pathway. J Cell Sci 116(Pt 20):4053-4056.

35. Kang R, Zeh HJ, Lotze MT, \& Tang D (2011) The Beclin 1 network regulates autophagy and apoptosis. Cell Death Differ 18(4):571-580.

36. Pattingre $\mathrm{S}$, et al. (2005) Bcl-2 antiapoptotic proteins inhibit Beclin 1-dependent autophagy. Cell 122(6):927-939.

37. Decuypere JP, Parys JB, \& Bultynck G (2012) Regulation of the autophagic bcl-2/beclin 1 interaction. Cells 1(3):284-312.

38. Sheng R \& Qin ZH (2015) The divergent roles of autophagy in ischemia and preconditioning. Acta Pharmacol Sin 36(4):411-420.

\section{Figures}

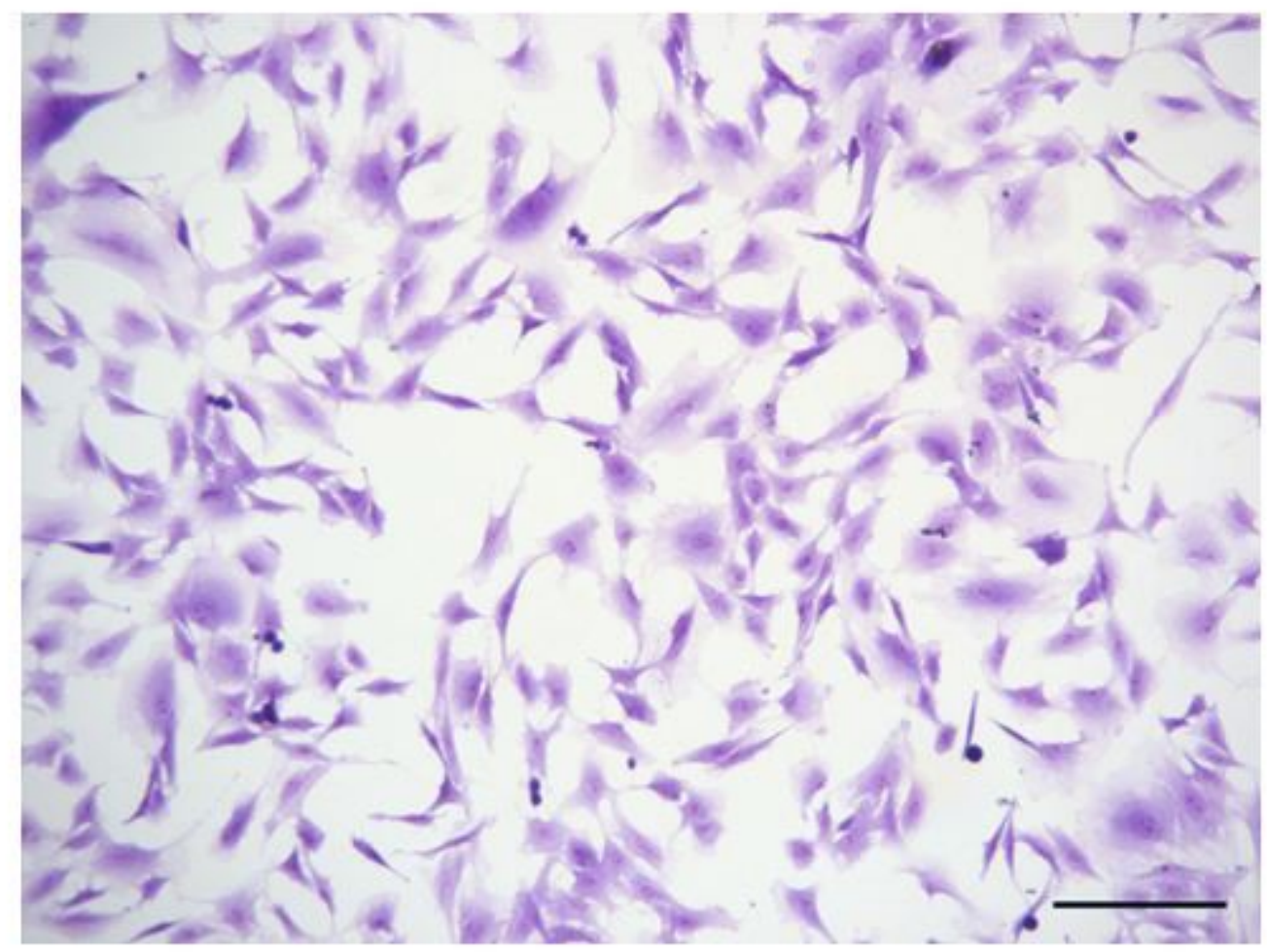

\section{Figure 1}


Bluish violet staining of isolated rat intervertebral disc cells. The isolated rat intervertebral disc cells were stained with bluish violet dye and observed under light microscopy. The cells were dyed blue-purple and presented as irregular polygons and some cells had long pseudopods. Scale bar $=100 \mu \mathrm{m}$.

A

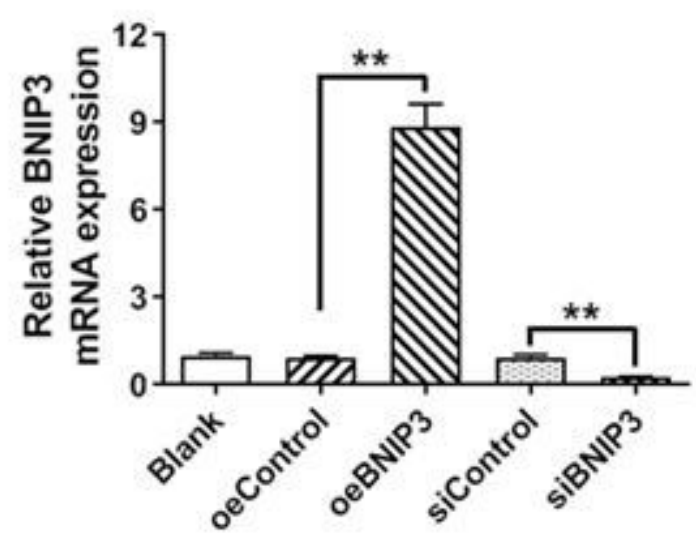

B

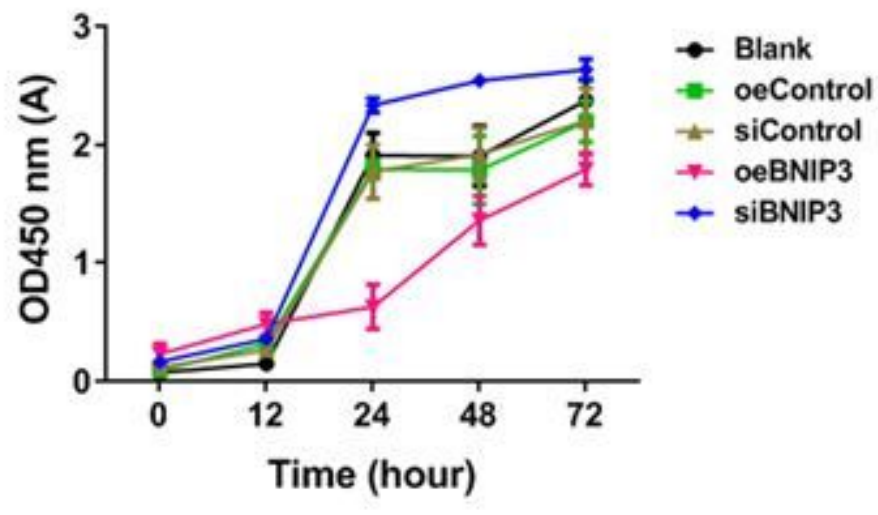

Figure 2

The overexpression of BNIP3 decreased the viability of rat intervertebral disc cells. (A). The isolated rat intervertebral disc cells were transfected with BNIP3 overexpression vector and control vector, BNIP3 interference vector and control vector, 48h after transfected, the BNIP3 expression level in different groups were detected by RT-PCR. The differences of BNIP3 mRNA expression were analyzed by 2- $\triangle \triangle \mathrm{CT}$ method. The BNIP3 expression in the samples was normalized against the expression of GAPDH, and gene expression in untreated cells (blank group) was normalized to 1. (B). The cells were placed on the 6-well plates and transfected with different BNIP3 vectors. The cell viability of in different groups was detected by CCK-8 method. Bars represent the mean \pm SD from three independent experiments. ${ }^{*} \mathrm{P}<0.01$ versus control by using unpaired two-tailed Student's $t$ test. 

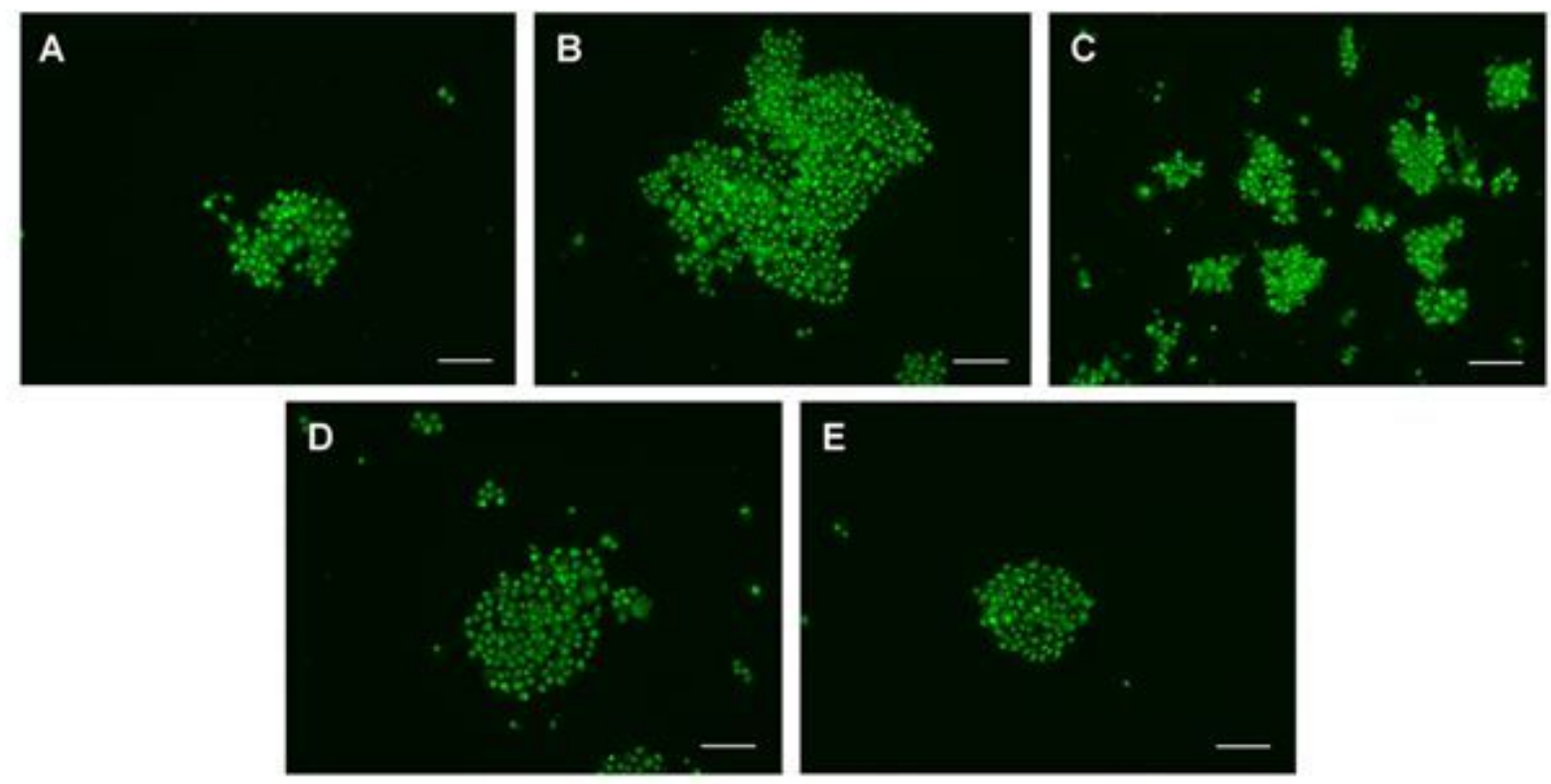

\section{Figure 3}

The autophagy of rat intervertebral disc cells in different groups was detected by acridine orange staining. (A). normal rat intervertebral disc cells (The blank group). (B). rat intervertebral disc cells transfected with overexpression empty vector (oeControl group). (C). rat intervertebral disc cells transfected with BNIP3 overexpression vector (oeBNIP3 group). (D). rat intervertebral disc cells transfected with RNAi empty vector (siControl group). (E). rat intervertebral disc cells transfected with BNIP3 interference vector (siBNIP3 group). The stained cells were observed by light microscopy, the scale bar $=100 \mu \mathrm{m}$. 

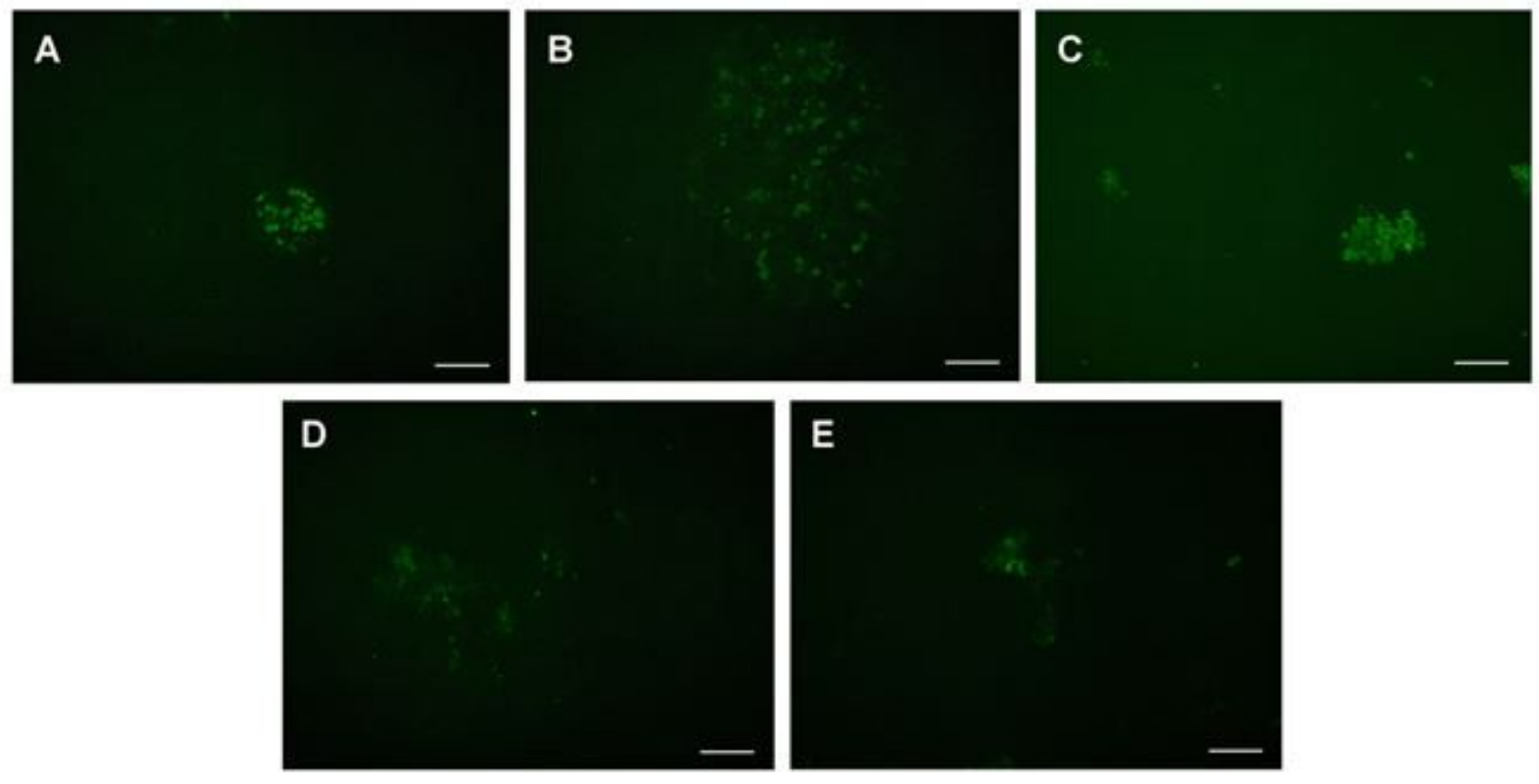

\section{Figure 4}

The autophagy of rat intervertebral disc cells in different groups was detected by MDC staining. (A). normal rat intervertebral disc cells (The blank group). (B). rat intervertebral disc cells transfected with overexpression empty vector (overexpression control group). (C). rat intervertebral disc cells transfected with BNIP3 overexpression vector (BNIP3 overexpression group). (D). rat intervertebral disc cells transfected with RNAi empty vector (interference control group). (E). rat intervertebral disc cells transfected with BNIP3 interference vector (BNIP3 interference group). The stained cells were observed by light microscopy, the scale bar $=100 \mu \mathrm{m}$. 
A

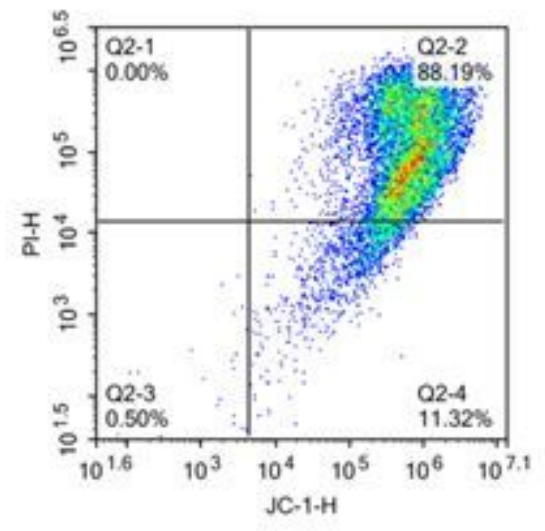

D

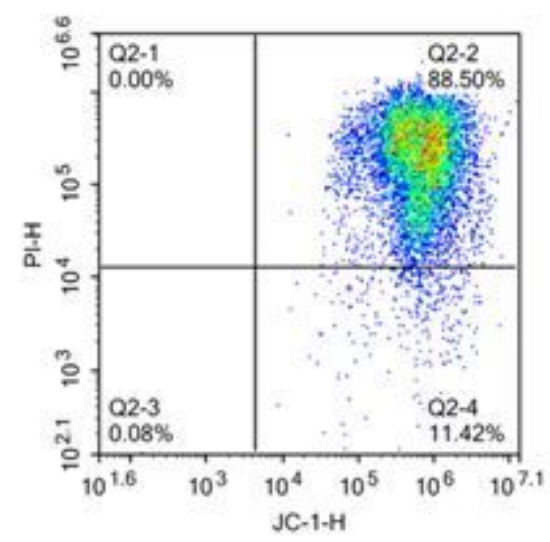

B

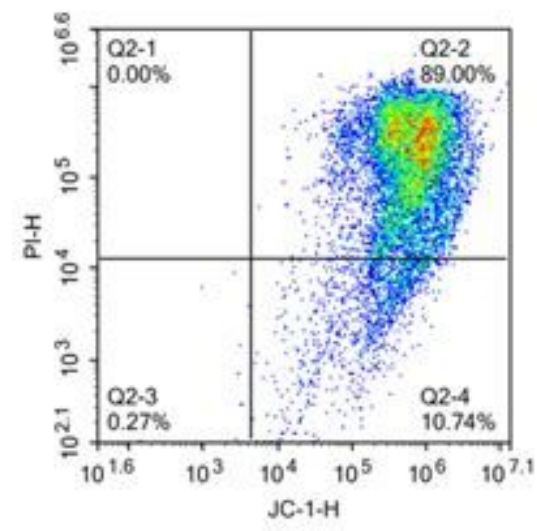

C

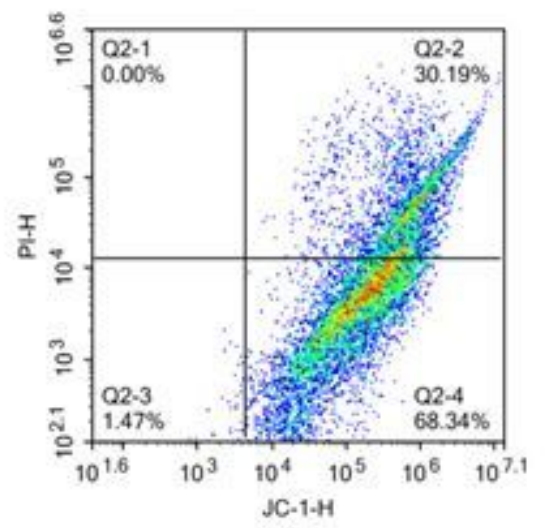

E

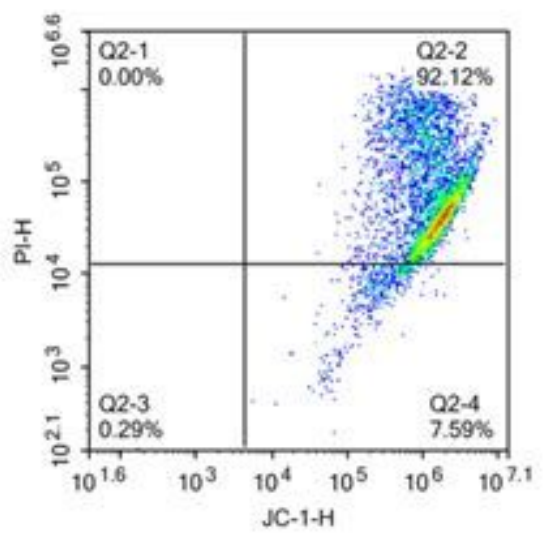

\section{Figure 5}

The cell membrane potential of rat intervertebral disc cells in different treatment groups was detected by flow cytometry. (A). normal rat intervertebral disc cells (The blank group). (B). rat intervertebral disc cells transfected with overexpression empty vector (overexpression control group). (C). rat intervertebral disc cells transfected with BNIP3 overexpression vector (BNIP3 overexpression group). (D). rat intervertebral disc cells transfected with RNAi empty vector (interference control group). (E). rat intervertebral disc cells transfected with BNIP3 interference vector (BNIP3 interference group). 
A

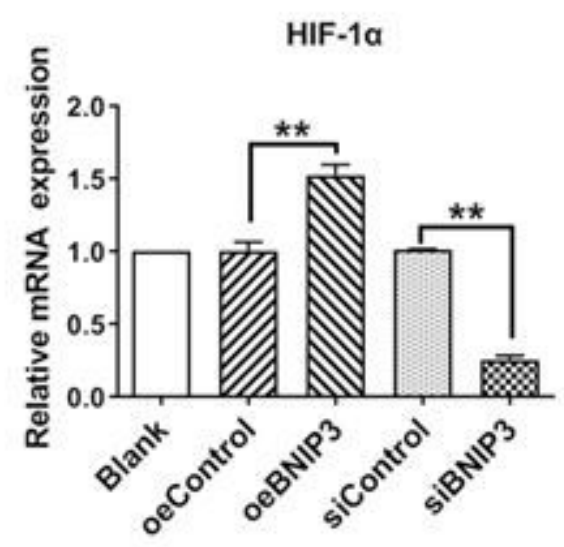

C

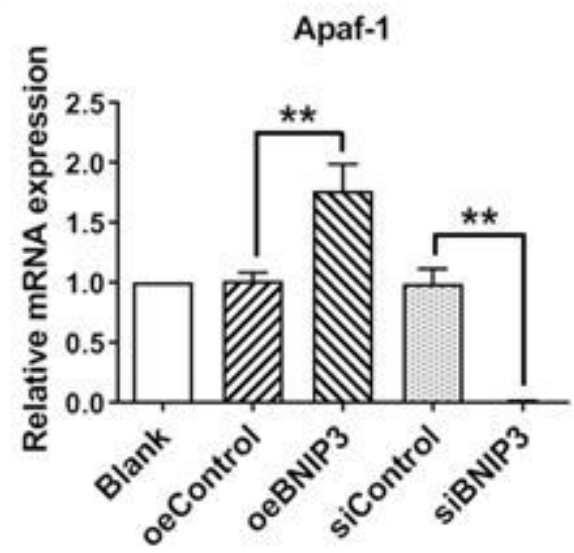

E

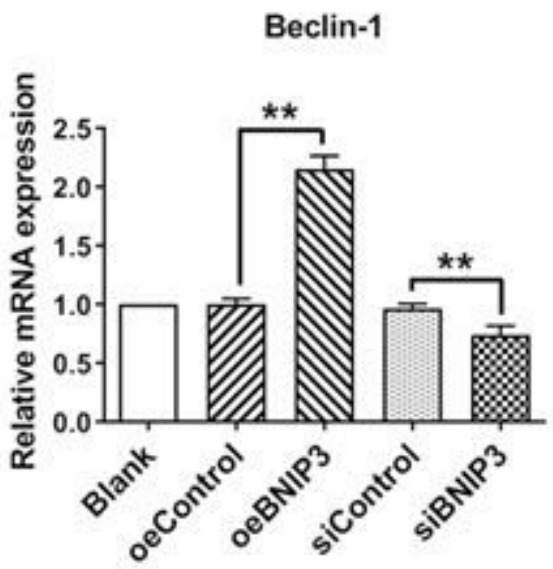

B

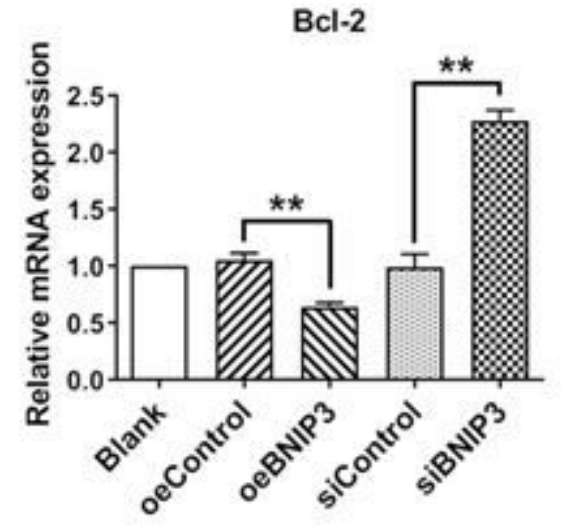

D

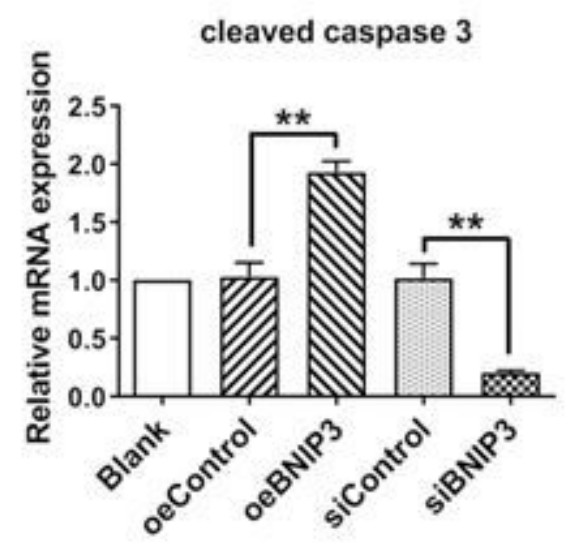

$\mathbf{F}$

LC-3

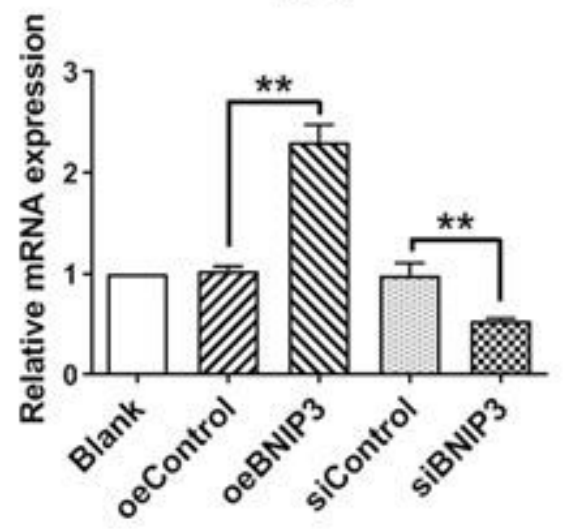

Figure 6

The expression differences of HIF-1a, Bcl-2, Apaf-1, cleaved caspase 3, Beclin-1 and LC-3 mRNA of rat intervertebral disc cells in different treatment groups were detected by RT-PCR. The expression differences of HIF-1 a (A), Bcl-2 (B), Apaf-1 (C), cleaved caspase 3 (D), Beclin-1 (E) and LC-3 (F) mRNA were showed and analyzed by 2- $\triangle \triangle C T$ method. The above mRNA expressions in the samples was normalized against the expression of GAPDH, and the mRNA expression in untreated cells (blank group) was normalized to 1. 
Bars represent the mean \pm SD from three independent experiments. ${ }^{*} \mathrm{P}<0.01$ versus control by using unpaired two-tailed Student's $t$ test.

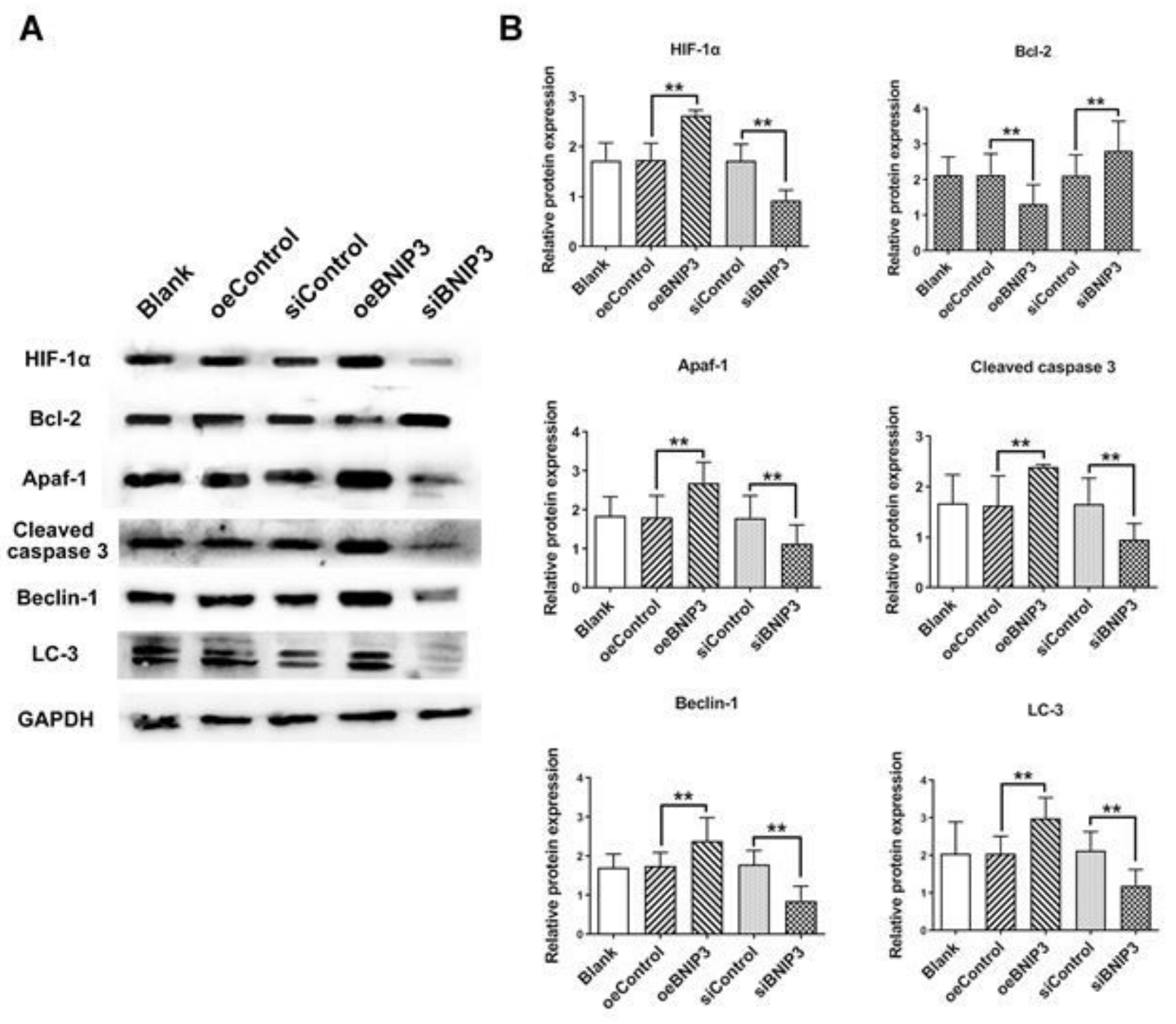

\section{Figure 7}

The expression differences of HIF-1a, Bcl-2, Apaf-1, cleaved caspase 3, Beclin-1 and LC-3 proteins of rat intervertebral disc cells in different treatment groups were detected by western blotting. (A). After the rat intervertebral disc cells transfected with BNIP3 overexpression or BNIP3 interference vectors, the expression of HIF-1a, Bcl-2, Apaf-1, cleaved caspase 3, Beclin-1 and LC-3 proteins in the cells were detected by western blotting. (B). The band intensities of the above proteins was quantitative analyzed according to their gray by Image $\mathrm{J}$ software. The gray analysis results were showed on the bottom panel. Bars represent the mean \pm SD from three independent experiments. ${ }^{\star \star} \mathrm{P}<0.01$ versus control by using unpaired two-tailed Student's t test. 